\title{
The Future of Parallel Tumor and Germline Genetic Testing: Is There a Role for All Patients With Cancer?
}

\author{
Ying L. Liu, MD, MPH, ${ }^{1}$ and Zsofia K. Stadler, MD ${ }^{1}$
}

\begin{abstract}
Under the traditional paradigm of genetic testing in cancer, the role of germline testing was to assess for the inherited risk of cancer, whereas the role of tumor testing was to determine therapeutic selection. Parallel tumor-normal genetic testing uses simultaneous genetic testing of the tumor and normal tissue to identify mutations and allows their classification as either germline or somatic. The increasing adoption of parallel testing has revealed a greater number of germline findings in patients who otherwise would not have met clinical criteria for testing. This result has widespread implications for the screening and further testing of at-risk relatives and for gene discovery. It has also revealed the importance of germline testing in therapeutic actionability. Herein, we describe the pros and cons of tumor-only versus parallel tumor-normal testing and summarize the data on the prevalence of incidental actionable germline findings. Because germline testing in patients with cancer continues to expand, it is imperative that systems be in place for the proper interpretation, dissemination, and counseling for patients and at-risk relatives. We also review new therapeutic approvals with germline indications and highlight the increasing importance of germline testing in selecting therapies. Because recommendations for universal genetic testing are increasing in multiple cancer types and the number of approved therapies with germline indications is also increasing, a gradual transition toward parallel tumor-normal genetic testing in all patients with cancer is foreseeable.
\end{abstract}

J Natl Compr Canc Netw 2021;19(7):871-878 doi: 10.6004/jnccn.2021.7044

${ }^{1}$ Clinical Genetics Service, Department of Medicine, Memorial Sloan Kettering Cancer Center, New York, New York.

\section{Historical Approaches to Genetic Testing in Cancer}

Although the inherited component of cancer susceptibility has been appreciated for decades, novel gene discoveries and new implications for therapeutics have highlighted its importance in modern cancer care. ${ }^{1}$ Traditionally, germline genetic testing involved a detailed review of personal and family cancer history with subsequent phenotype-driven genetic testing, often performed sequentially with 1 or 2 genes at a time. The primary purpose of germline genetic testing was to identify those with an inherited risk for cancer who could then benefit from tailored cancer screening/risk-reducing measures and cascade testing of at-risk family members., ${ }^{2,3}$ The decreasing costs of next-generation sequencing technologies and the widespread use of multigene panels have improved accessibility to testing and facilitated the discovery of novel gene-cancer associations and the incorporation of genes with varied cancer risk estimates from low to high penetrance. ${ }^{3}$ In addition, the increasing number of cancer susceptibility genes that simultaneously drive cancer risk and may direct cancer therapy, such as BRCA1/2 mutations in hereditary breast and ovarian syndrome, ${ }^{4}$ has placed increasing importance on genetic testing in cancer care. ${ }^{1}$ As a result, more patients with cancer are undergoing genetic testing, and there is a drive for universal germline testing for all patients with cancer. ${ }^{5}$

Similarly, in the era of precision medicine, the scope of tumor testing has also expanded, driven by the discovery of novel genotype-directed targeted therapies. ${ }^{6,7}$ Whereas tumor sequencing was traditionally performed in limited situations and often only in patients with advanced or metastatic cancer for genotype-directed clinical trial eligibility, it is now expanding into other clinical scenarios as treatment implications become more widespread. ${ }^{8}$ The recent NCI-MATCH study showed not only the feasibility of large-scale, pan-cancer tumor testing for the identification of clinically actionable mutations, but also the ability to assign patients to genotypedirected treatment in a clinical or research setting. ${ }^{9}$ Although germline genetic testing and tumor somatic testing were traditionally performed independently, novel 
parallel sequencing technologies have juxtaposed the 2 processes with widespread implications. ${ }^{10}$ Herein, we discuss the pros and cons of tumor-only versus parallel tumor-normal testing and review the implications and future of parallel testing.

\section{Tumor-Only Versus Parallel Tumor- Normal Testing}

The 2 approaches to the genomic testing of tumors include (1) the sequencing of tumor tissue only, wherein the tumor sequence analysis includes both somatic and germline alterations (tumor); and (2) the sequencing of tumor and matched normal tissue with the subsequent subtraction of germline alterations from somatic alterations, resulting in the identification of somatic mutations only on tumor assessment (somatic). ${ }^{11}$ Notably, although germline sequencing occurs in both the tumor-only and the tumor-normal matched approach, in the former, the germline assessment is an indirect result of the tumor sequencing and in the tumor-normal analysis, the germline is directly interrogated. The parallel tumor-normal matched approach allows for a direct differentiation of somatic versus germline findings and the potential for the return of germline-specific genetic test results to the patient (Figure 1). ${ }^{10,12,13}$

The advantage of a tumor-only approach is efficiency, because germline DNA is not directly interrogated, and the necessary informed consents, privacy protections, and pretesting/posttesting genetic counseling inherent to traditional germline genetic testing are not routinely needed..$^{14,15}$ For the identification of therapeutically actionable genetic mutations, knowledge of the origin of the mutation as somatic versus germline is not always necessary. In addition, novel computational algorithms may eventually facilitate the accurate identification of somatic versus germline variants from tumor testing alone, raising the possibility of the return of germline results in the context of appropriate pretest informed consent. ${ }^{16}$

The disadvantages include challenges to the interpretation of results and the need for additional germline testing in certain patients, which could lead to delays in management and cascade testing. For example, tumoronly testing of a breast cancer sample may identify a $B R C A 1 / 2$ mutation, but it is unclear whether this mutation is somatic or germline. Additional genetic testing would be necessary but could potentially delay decisions regarding surgical management (mastectomy vs lumpectomy) and notification of at-risk relatives. Notably, negative tumor-only testing is not an adequate surrogate for dedicated germline testing, especially when the implications for cancer surveillance, treatment, and cascade testing are so critical. As recently shown by Lincoln et $\mathrm{al}^{17} 8.1 \%$ of pathogenic germline mutations were missed in patients who previously underwent tumor-only sequencing

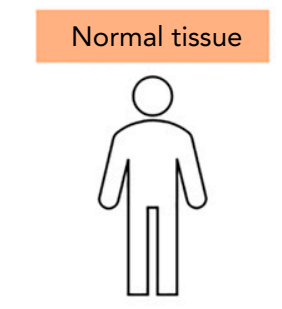

DDDDX

CGGTACCGAT

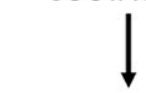

Germline result

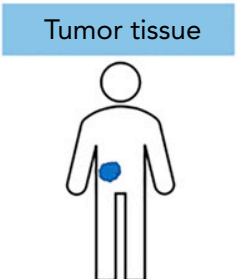

DODDD

CGTTACCGAT

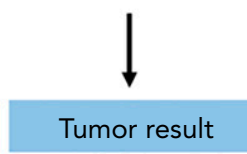

Paired normal and tumor

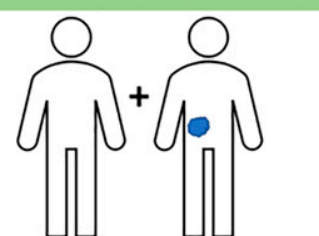

DODDOC DDODD

CGGTACCGAT CGTTACCGAT

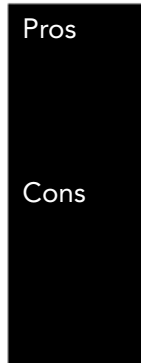

\begin{tabular}{l} 
- Assess inherited risk \\
- Cancer screening \\
- Counseling for families \\
- Reproductive planning \\
\hline - Limited to patients who meet \\
guidelines \\
- Testing interpretation difficulties \\
(eg. clonal hematopoiesis \\
and mosaicism)
\end{tabular}

- Inform diagnosis and prognosis

- Guide therapeutic decisions for targeted therapies

- Assess MSI for immunotherapies

- Inability to distinguish somatic vs germline variants.

- Inadequate surrogate for direct germline testing

- Need for further genetic testing and potential delays in care

Figure 1. Pros and cons of tumor-only versus paired tumor-normal genetic testing. This figure depicts the results of germline, tumor, and paired tumor-normal sequencing and displays how paired sequencing allows the differentiation of germline (red) versus somatic (blue) results. Abbreviation: MSI, microsatellite instability. 
because of the technical limitations of tumor sequencing, variant interpretation differences, or differences in the genes tested in the tumor versus the germline.

Perhaps most importantly, in tumor-only testing, the germline nature of findings may be misinterpreted by providers inexperienced in cancer genetics or incorrectly relayed to patients, resulting in suboptimal care not only for the patient but also for at-risk family members. ${ }^{18}$ As a result, the American College of Medical Genetics and Genomics ${ }^{19}$ and $\mathrm{NCCN}^{20}$ have issued guidelines for the reporting of potential germline variants from tumor testing, and laboratories and clinicians should receive guidance and training on interpreting tumor-only testing and potential germline findings. ${ }^{21,22}$ Even in tumor-only testing, multidisciplinary care is often necessary to identify potential germline variants and facilitate genetic counseling and confirmatory germline testing. ${ }^{19,21-23}$

The advantage of parallel tumor-normal testing is that it provides an unbiased assessment of the germline and somatic contribution to cancer risk and development. ${ }^{10}$ It also allows the integration of tumor and normal genomic data and complex interpretation, including the evaluation of loss of heterozygosity in tumor tissues and the differentiation of mosaicism and clonal hematopoiesis, which have implications for the heritability of findings. ${ }^{10}$ From a technical standpoint, it allows sample verification and additional quality control checks and may provide insight into the classification of germline variants. ${ }^{24}$

Disadvantages include the extra costs, resources, and expertise that are necessary for the successful implementation, interpretation, and dissemination of results. There also need to be mechanisms in place for the timely and comprehensive management of any germline findings. In addition, the identification of "incidental" germline findings may be the double-edged sword of parallel tumornormal testing. Although such germline findings may better elucidate cancer risk in families, the uncertainty of cancer risk estimates or gene penetrance in families without the corresponding phenotypic features make management decisions difficult, particularly in situations where risk-reducing surgeries may be relevant. For example, in a patient without a family history of gastric or lobular breast cancer, an incidental finding of a $\mathrm{CDHl}$ germline mutation on parallel testing makes decisions regarding prophylactic gastrectomy extremely difficult. ${ }^{25,26}$

\section{Parallel Testing Reveals Incidental Actionable Germline Findings}

With the advent of tumor-normal testing, more clinically actionable germline findings are being discovered across cancer types, often in patients who do not meet traditional criteria for genetic testing. ${ }^{27}$ In a study of 1,566 patients with paired tumor-normal testing using a 341gene panel (MSK IMPACT), ${ }^{6} 246$ patients (15.7\%) had at least 1 pathogenic or likely pathogenic variant in 1 of 187 Mendelian disease-associated genes. ${ }^{28} \mathrm{~A}$ subsequent study examined 10,336 patients who underwent tumor-normal sequencing with MSK IMPACT, of which 1,040 were consented for germline analysis including 76 cancerpredisposing genes. Of these patients, 182 (17.5\%) had clinically actionable variants conferring cancer susceptibility. Notably, 101, or $55 \%$ of germline-positive patients, would not have met the clinical criteria for guidelinebased genetic testing. ${ }^{29}$ Two smaller studies found that rates of discovery of pathogenic germline variants in patients with paired tumor-normal genetic testing were slightly lower, ranging from $2.3 \%$ to $4.3 \%{ }^{30,31}$ In the largest study, Huang et $\mathrm{al}^{32}$ used an automatic germline variant classification pipeline (CharGer) to analyze 10,389 patients who underwent tumor-normal testing from The Cancer Genome Atlas and found that 853 patients $(8 \%)$ had pathogenic or likely pathogenic germline variants (Table 1).

\section{New Opportunities for Gene Discovery and Novel Cancer Predisposition Syndromes}

Parallel sequencing provides opportunities for the discovery of novel genes and predisposition syndromes in many new cancer types. In a study of 451 patients with locally advanced or metastatic prostate cancer who underwent parallel sequencing, germline findings were available in a subset of 221 patients, of whom 42 (19\%) had pathogenic/ likely pathogenic germline mutations in DNA damage repair genes. ${ }^{33}$ In a study of 254 patients with renal cell carcinoma who underwent paired sequencing, 41 (16.1\%) had germline mutations. Several of these patients $(n=5)$ would not have met the clinical guidelines for genetic testing. In addition, an overrepresentation of CHEK2 mutations was seen, suggesting a potentially novel association. ${ }^{34}$ A similar study conducted in 586 patients with urothelial cancers, which are traditionally associated with mismatch repair deficiency (MMR-D) and Lynch syndrome, identified 54 individuals $(9.2 \%)$ with pathogenic/ likely pathogenic germline variants in DNA repair genes, excluding MMR genes. ${ }^{35}$ Increasing repositories of patients with pan-cancers and paired tumor-normal testing have also allowed the discovery of novel cancer predisposition syndromes, particularly in individuals with multiple primary tumors. ${ }^{36,37}$

Parallel testing also highlights the need for wider germline genetic testing in cancer types not traditionally associated with hereditary cancer syndromes. A study of tumor-normal sequencing in multiple samples from 88 patients with metastatic neuroendocrine tumors found that 14 patients (16\%) had clinically actionable germline variants, suggesting a need for universal germline testing in this rare tumor type.$^{38}$ In a study of 15,045 patients who underwent paired testing to determine microsatellite 


\begin{tabular}{|c|c|c|}
\hline References & Study Design & Findings \\
\hline Schrader et $\mathrm{al}^{28}$ & $\begin{array}{l}\text { 1,566 patients with tumor-normal testing in a } 341 \text {-gene } \\
\text { panel (MSKCC IMPACT) - } 187 \text { genes with Mendelian } \\
\text { disease associations }\end{array}$ & $\begin{array}{l}246 \text { patients }(15.7 \%) \text { had presumed pathogenic germline variants } \\
\text { in known Mendelian disease-associated genes }\end{array}$ \\
\hline Meric-Bernstam et al ${ }^{30}$ & 1,000 patients with tumor-normal testing of 202 genes & $\begin{array}{l}43 \text { patients }(4.3 \%) \text { had likely pathogenic germline variants in } 19 \\
\text { genes considered actionable; } 2.3 \% \text { of patients had previously } \\
\text { unrecognized germline pathogenic mutations }\end{array}$ \\
\hline Seifert et $\mathrm{al}^{31}$ & $\begin{array}{l}439 \text { patients who underwent tumor-normal sequencing- } \\
36 \text { hereditary cancer susceptibility genes }\end{array}$ & $\begin{array}{l}19 \text { patients }(4.3 \%) \text { had germline variants indicative of hereditary } \\
\text { cancer predisposition; for } 10 / 19 \text { patients, these findings were } \\
\text { new diagnostic information }\end{array}$ \\
\hline Mandelker et $\mathrm{a}^{29}$ & $\begin{array}{l}\text { 10,336 patients with tumor-normal testing (MSKCC } \\
\text { IMPACT) - } 1,040 \text { patients with reported germline results } \\
\text { from a panel of } 76 \text { genes }\end{array}$ & $\begin{array}{l}182 \text { patients }(17.5 \%) \text { had clinically actionable mutations; } 101 \\
(9.7 \%) \text { of these patients would not have met clinical criteria for } \\
\text { germline testing }\end{array}$ \\
\hline Huang et $\mathrm{a}^{32}$ & $\begin{array}{l}\text { 10,389 patients who underwent tumor-normal testing; } \\
\text { used automatic germline variant classification pipeline }\end{array}$ & $\begin{array}{l}853 \text { patients ( } 8 \% \text { ) had pathogenic or likely pathogenic germline } \\
\text { variants }\end{array}$ \\
\hline
\end{tabular}

instability (MSI) status, Lynch syndrome was identified in $16.3 \%$ of MSI-high (MSI-H) tumors, $1.9 \%$ of MSIindeterminate tumors, and $0.3 \%$ of microsatellite stable (MSS) tumors. Among the patients with Lynch syndrome, $50 \%$ had tumors other than classic colorectal or endometrial cancers, and $45 \%$ of these patients did not meet the clinical criteria for testing. This finding suggests that all patients with MSI-H or MMR-D tumors should undergo genetic evaluation for Lynch syndrome, and highlights the utility of integrating somatic and germline findings. ${ }^{39}$ In addition, studies of patients with exocrine pancreatic cancer who underwent parallel tumor-normal genetic testing have found high rates of pathogenic germline alterations ranging from $15.1 \%$ to $19.8 \%{ }^{40,41}$ In fact, genomic alterations in exocrine pancreatic cancers are more likely to be germline than somatic,,$^{40}$ and many are potentially clinically actionable. ${ }^{42}$ Accordingly, the recent NCCN Clinical Practice Guidelines in Oncology (NCCN Guidelines) for Genetic/ Familial High-Risk Assessment: Breast, Ovarian, and Pancreatic have recommended germline testing in all patients with pancreatic cancer..$^{20}$

\section{Limitations of Parallel Testing}

Although these studies are compelling and show that a large proportion of patients may benefit from universal tumor-normal genetic testing, there are limitations and challenges to parallel testing. Many of these studies were conducted at large, tertiary cancer centers and are subject to ascertainment bias, potentially inflating the rates of germline findings. In addition, it is unclear whether incidental germline findings in tumor types not traditionally associated with hereditary cancer syndromes are true drivers of disease or are merely passenger mutations. Indeed, especially common tumors such as lung, breast, prostate, and colorectal cancer can occur in individuals with germline mutations in cancer susceptibility genes in a sporadic fashion. Jonsson et $\mathrm{al}^{43}$ examined BRCAl/2 mutations across pan-cancers and found that the role of these mutations in driving cancer phenotypes was dependent on tumor lineage and called into question the utility of clinical decisions based on these mutations. Latham et $\mathrm{al}^{39}$ also found that a small proportion of patients with Lynch syndrome with germline mutations harbored tumors that were MSS and did not exhibit an MMR-D signature, suggesting that the germline mutations may be incidental. However, these studies also highlight the need to obtain both tumor and germline sequencing to fully understand the biology of tumors and separate the true drivers of disease from passenger mutations.

Further limiting the universal adoption of parallel testing are difficulties in the interpretation of "normal" tissue when sequencing blood due to clonal hematopoiesis $(\mathrm{CH})$ and mosaicism, which can confound germline results. $\mathrm{CH}$ refers to somatic mutations in the hematopoietic lineage that can be identified through the sequencing of blood for normal tissue, and mosaicism refers to differential cell populations with distinct genotypes within one individual. Although genetic variants associated with $\mathrm{CH}$ or mosaicism should be present at lower variant fractions than the traditional $50 \%$ of germline heterozygous mutations, technical challenges in sequencing and early mosaicism events can make interpretation challenging. ${ }^{44,45}$

In addition, many centers may not have an existing genetic counseling infrastructure to provide a comprehensive discussion of the risks/benefits of genetic testing and a timely dissemination of sensitive germline results and notification of at-risk relatives. ${ }^{46}$ Results from the MAGENTA (Making GENetic Testing Accessible) study found that electronic genetic education and the release of results without traditional genetic counseling was noninferior with regard to patient distress and was associated with higher test completion rates, suggesting that a paradigm of individualized genetic counseling for patients 
with positive findings alone may be acceptable. ${ }^{47}$ There are also challenges related to informed consent, privacy protection, and future research regarding these germline specimens and data generated through parallel testing. ${ }^{48,49}$ Given these challenges, working groups have been formed to discuss best practices for the integration of somatic and germline data to facilitate the interpretation and application of data. ${ }^{24}$

\section{Germline Findings Can Lead to Novel Therapeutic Implications}

Despite these limitations and challenges, the necessity of parallel tumor-normal testing is becoming more apparent as the actionability of germline findings expands with an increasing number of FDA-approved therapeutics with germline indications (Table 2). Early studies of targeted therapies for germline indications were conducted in basal cell carcinomas ${ }^{50}$ and thyroid cancers. ${ }^{51}$ Both studies included a significant number of patients with known genetic cancer predisposition syndromes-Gorlin syndrome and multiple endocrine neoplasia 2 syndrome-and found efficacy in those populations. Recently, another RET inhibitor, selpercatinib, was approved for RET-mutant medullary thyroid cancers. ${ }^{52}$ Targeted therapies, including everolimus and selumetinib, have also been studied in rare, genetic conditions associated with multiple cancer types, such as tuberous sclerosis and neurofibromatosis $1 . .^{53-55}$

Subsequently, a study of the PD-1 inhibitor pembrolizumab in patients with advanced/metastatic solid tumors with MMR-D who experienced disease progression on traditional chemotherapy found efficacy in this population. The study included many patients with Lynch syndrome with tumors exhibiting MMR-D, the hallmark of Lynch syndrome-associated cancers. ${ }^{56}$ As a result, pembrolizumab received the first tumor-agnostic indication from the FDA for all MMR-D/MSI-H tumors, including those in patients with Lynch syndrome.

More recently, PARP inhibitors have gained multiple germline-based FDA approvals in a variety of tumor types. In women with ovarian cancer and germline $B R C A$ mutations, the PARP inhibitor olaparib was shown to be effective as a treatment in the recurrent setting and as maintenance in the up-front setting. ${ }^{57,58}$ Subsequently, multiple PARP inhibitors have been approved in ovarian cancer for a wider population of women, including those with somatic BRCA mutations, ${ }^{59}$ those with homologous repair-deficient (HRD) tumors, ${ }^{60,61}$ and in all patients as maintenance therapy. ${ }^{62}$ PARP inhibitors are also approved in metastatic breast cancer for patients with germline BRCA1/2 mutations. ${ }^{63,64}$ Interestingly, in metastatic prostate cancer, rucaparib is approved for patients with BRCA1/ 2 mutations ${ }^{33,65}$; however, olaparib is approved in those with a wider range of germline HRD genes. ${ }^{66,67}$ Olaparib was also recently approved in patients with metastatic pancreatic cancer and germline BRCA1/2 mutations. ${ }^{42}$ These studies suggest that knowledge of germline mutations, particularly those in BRCA1/2 or other HRD genes, may influence the effectiveness of PARP inhibitors and affect clinical management.

\begin{tabular}{|c|c|c|c|}
\hline Cancer Type & Drug & Germline Variant & Evidence \\
\hline \multirow[t]{2}{*}{ Breast cancer } & Olaparib & BRCA1/2 & OLYMPIAD (Robson et al ${ }^{64}$ ) \\
\hline & Talazoparib & BRCA1/2 & EMBRACA (Litton et $\mathrm{al}^{63}$ ) \\
\hline \multirow[t]{3}{*}{ Ovarian cancer } & Olaparib & $B R C A 1 / 2$ & Study 42 (Domchek et $\mathrm{al}^{67}$ ) and SOLO1 (Moore et al ${ }^{58}$ ) \\
\hline & Rucaparib & $B R C A 1 / 2$ & Study 10/ARIEL2 (Swisher et al ${ }^{59}$ ) \\
\hline & Niraparib & BRCA1/2 & QUADRA (Moore et $\mathrm{al}^{61}$ ) \\
\hline \multirow[t]{2}{*}{ Metastatic prostate cancer } & Olaparib & HR genes & PROfound (de Bono et $\mathrm{al}^{66}$ ) \\
\hline & Rucaparib & $B R C A 1 / 2$ & TRITON2 (Abida et $a^{65}$ ) \\
\hline Pancreatic cancer & Olaparib & BRCA1/2 & POLO (Golan et $\mathrm{al}^{42}$ ) \\
\hline Advanced/Metastatic solid tumors & Pembrolizumab & MMR-D (Lynch syndrome) & KEYNOTE 016 (Le et al ${ }^{56}$ ) \\
\hline Basal cell carcinoma & Vismodegib & PTCH1 (Gorlin syndrome) & ClinicalTrials.gov identifier: NCT00833417 (Sekulic et al $\left.{ }^{50}\right)$ \\
\hline \multirow[t]{2}{*}{ Thyroid cancer } & Vandetanib & RET (MEN2) & ZETA (Wells et al ${ }^{51}$ ) \\
\hline & Selpercatinib & RET (MEN2) & LIBRETTO (Wirth et $\mathrm{al}^{52}$ ) \\
\hline Subependymal giant-cell astrocytoma & Everolimus & TSC1/2 (tuberous sclerosis) & EXIST-1 (Krueger et al ${ }^{55}$ ) \\
\hline Renal angiomyolipoma & Everolimus & TSC1/2 (tuberous sclerosis) & EXIST-2 (Bissler et al ${ }^{53}$ ) \\
\hline Plexiform neurofibromas & Selumetinib & NF1 (neurofibromatosis type 1) & ClinicalTrials.gov identifier: NCT01362803 (Dombi et al ${ }^{54}$ ) \\
\hline
\end{tabular}

Abbreviations: HR, homologous repair; MEN, multiple endocrine neoplasia; MMR-D, mismatch repair-deficient. 


\section{Future of Parallel Testing: Informing Treatment Decisions}

As the number of germline indications for therapies-both FDA-approved and for clinical trials-increases, germline results from parallel tumor-normal testing are directly impacting treatment decisions. ${ }^{68}$ In a study of 11,947 patients who underwent parallel tumor-normal testing with MSK IMPACT, 1,042 (9\%) had a likely pathogenic/ pathogenic germline alteration in a gene with a targeted therapy that was FDA-approved or had supporting data of such quality that it might warrant future approval. Most mutations were in BRCA1/2, ATM, or Lynch genes, and the most common cancer types represented were ovarian, breast, pancreatic, and prostate cancers. Notably, among the subset of patients with advanced cancer, $40 \%$ received germline-directed treatment. Common targeted therapies included PARP and checkpoint inhibitors. ${ }^{69}$ These studies highlight the importance of parallel testing in expanding the population of patients receiving germline genetic information to not only improve the assessment of inherited risk but also expand therapeutic options.

\section{Expanding Germline Testing to All Cancer Types?}

Given the increasing therapeutic implications of germline findings, there have been major updates to recommendations for genetic testing in various cancer types. ${ }^{70}$ Although universal germline testing for $B R C A$ mutations had been recommended for ovarian cancer, this recommendation has now expanded to patients with metastatic breast cancer, metastatic prostate cancer, and pancreatic cancer. In addition, there are expanded recommendations for MMR staining and MSI testing in all patients with colorectal and endometrial cancer. As germline testing expands across tumor types, there is a question of whether patients with earlier-stage, nonmetastatic cancers should undergo germline testing because targeted therapies are moving into earlier stages of disease. For example, there are ongoing adjuvant studies of checkpoint inhibitors in patients with Lynch syndrome with endometrial cancer (GY020; ClinicalTrials.gov identifier: NCT04214067) and colon cancer (NCT02912559) and adjuvant studies of PARPi in women with breast cancer and germline BRCA mutations (OlympiA; NCT02032823). Preliminary data also support the efficacy of PARPi monotherapy as neoadjuvant treatment in women with germline $B R C A$ mutations and operable breast cancer, ${ }^{71}$ with an ongoing phase III trial (NCT03499353).

\section{Call to Action: Parallel Testing in All Patients With Cancer}

The integration of somatic and germline testing is critical to comprehensively characterize pathogenic germline mutations, accurately assess drivers of cancer risk, and make therapeutic decisions. Going forward, there may be a benefit to parallel next-generation sequencing tumor-normal testing for all patients with cancer. In addition, studies show that patients are very interested in paired testing and in obtaining germline results. ${ }^{72}$ As parallel testing becomes more common, there will be a need to carefully interpret results for both therapeutic potential and cancer predisposition risk. Challenges for moving forward include decisions on multigene, tumor-agnostic germline panels versus more disease-directed panels, and the need for innovative systems to provide the appropriate counseling and dissemination of results. However, expanded germline testing may continue to have unforeseen benefits, such as expanded opportunities for cascade testing, ${ }^{73}$ potentially benefiting not only patients but also their at-risk relatives.

Submitted September 13, 2020; final revision received December 28, 2020; accepted for publication April 9, 2021

Disclosures: Dr. Liu has disclosed receiving grant/research support from AstraZeneca and GlaxoSmithKline/Tesaro. Dr. Stadler has disclosed having an immediate family member who serves as a consultant in ophthalmology for Alcon, Adverum Biotechnologies, Allergan, Genentech/Roche, Novartis, Neurogene, Gyroscope Therapeutics, Optos, Regeneron, and RegenexBio.

Funding: This work was supported by funding from the NIH (MSKCC is supported by the $\mathrm{NCl}$ under Core Grant P30 CA008748).

Disclaimer: The content is solely the responsibility of the authors and does not necessarily represent the official views of the $\mathrm{NIH}$.

Correspondence: Zsofia K. Stadler, MD, Clinical Genetics Service, Department of Medicine, Memorial Sloan Kettering Cancer Center, 300 East 66th Street, 10th Floor, New York, NY 10065. Email: stadlerz@mskcc.org

\section{References}

1. Offit K. Decade in review-genomics: a decade of discovery in cancer genomics. Nat Rev Clin Oncol 2014;11:632-634.

2. Robson ME, Bradbury AR, Arun B, et al. American Society of Clinical Oncology policy statement update: genetic and genomic testing for cancer susceptibility. J Clin Oncol 2015;33:3660-3667.

3. Stadler ZK, Schrader KA, Vijai J, et al. Cancer genomics and inherited risk. J Clin Oncol 2014;32:687-698.

4. Chen S, Parmigiani G. Meta-analysis of BRCA1 and BRCA2 penetrance. J Clin Oncol 2007;25:1329-1333.

5. Samadder NJ, Riegert-Johnson D, Boardman L, et al. Comparison of universal genetic testing vs guideline-directed targeted testing for patients with hereditary cancer syndrome. JAMA Oncol 2021;7: 230-237.

6. Zehir A, Benayed R, Shah RH, et al. Mutational landscape of metastatic cancer revealed from prospective clinical sequencing of 10,000 patients. Nat Med 2017;23:703-713.

7. Collins FS, Varmus $\mathrm{H}$. A new initiative on precision medicine. $\mathrm{N}$ Engl J Med 2015;372:793-795.

8. Hyman DM, Taylor BS, Baselga J. Implementing genome-driven oncology. Cell 2017;168:584-599.

9. Flaherty KT, Gray RJ, Chen AP, et al. Molecular landscape and actionable alterations in a genomically guided cancer clinical trial: National Cancer 
Institute Molecular Analysis for Therapy Choice (NCI-MATCH). J Clin Oncol 2020;38:3883-3894.

10. Mandelker D, Ceyhan-Birsoy O. Evolving significance of tumor-normal sequencing in cancer care. Trends Cancer 2020;6:31-39.

11. Trottier AM, Cavalcante de Andrade Silva M, Li Z, et al. Somatic mutation panels: time to clear their names. Cancer Genet 2019;235:84-92.

12. Mandelker D. Toward concurrent testing for somatic and germline variants in cancer patients. Clin Cancer Res 2016;22:3987-3988.

13. Mandelker $D$, Zhang $L$. The emerging significance of secondary germline testing in cancer genomics. J Pathol 2018;244:610-615.

14. Giri VN, Yurgelun MB, Robson ME. The role of genetic counseling in familial and sporadic cancer: considerations, challenges, and collaboration. Ann Intern Med 2017;167:884-885.

15. Robson ME, Storm CD, Weitzel J, et al. American Society of Clinical Oncology policy statement update: genetic and genomic testing for cancer susceptibility. J Clin Oncol 2010;28:893-901.

16. Sun JX, He Y, Sanford E, et al. A computational approach to distinguish somatic vs. germline origin of genomic alterations from deep sequencing of cancer specimens without a matched normal. PLOS Comput Biol 2018;14:e1005965.

17. Lincoln SE, Nussbaum RL, Kurian AW, et al. Yield and utility of germline testing following tumor sequencing in patients with cancer. JAMA Netw Open 2020;3:e2019452

18. Vlessis K, Purington N, Chun N, et al. Germline testing for patients with BRCA1/2 mutations on somatic tumor testing. JNCl Cancer Spectr 2019;4:pkz095.

19. Li MM, Chao E, Esplin ED, et al. Points to consider for reporting of germline variation in patients undergoing tumor testing: a statement of the American College of Medical Genetics and Genomics (ACMG). Genet Med 2020;22:1142-1148.

20. Daly MB, Pal T, Berry MP, et al. NCCN Clinical Practice Guidelines in Oncology: Genetic/Familial High-Risk Assessment: Breast, Ovarian and Pancreatic. Version 2. 2021. Accessed May 12, 2021. To view the most recent version, visit NCCN. org

21. Raymond VM, Gray SW, Roychowdhury S, et al. Germline findings in tumor-only sequencing: points to consider for clinicians and laboratories. J Natl Cancer Inst 2015;108:djv351.

22. Mandelker D, Donoghue M, Talukdar S, et al. Germline-focussed analysis of tumour-only sequencing: recommendations from the ESMO Precision Medicine Working Group. Ann Oncol 2019;30:1221-1231.

23. Klek S, Heald B, Milinovich A, et al. Genetic counseling and germline testing in the era of tumor sequencing: a cohort study. JNCl Cancer Spectr 2020;4:pkaa018.

24. Walsh MF, Ritter DI, Kesserwan C, et al. Integrating somatic variant data and biomarkers for germline variant classification in cancer predisposition genes. Hum Mutat 2018;39:1542-1552.

25. Lowstuter K, Espenschied CR, Sturgeon D, et al. Unexpected CDH1 mutations identified on multigene panels pose clinical management challenges. JCO Precis Oncol 2017;1:1-12.

26. Vos EL, Salo-Mullen EE, Tang LH, et al. Indications for total gastrectomy in $\mathrm{CDH} 1$ mutation carriers and outcomes of risk-reducing minimally invasive and open gastrectomies. JAMA Surg 2020;155:1050-1057.

27. Bombard Y, Robson M, Offit K. Revealing the incidentalome when targeting the tumor genome. JAMA 2013;310:795-796.

28. Schrader KA, Cheng DT, Joseph V, et al. Germline variants in targeted tumor sequencing using matched normal DNA. JAMA Oncol 2016;2:104-111.

29. Mandelker $D$, Zhang $L$, Kemel $Y$, et al. Mutation detection in patients with advanced cancer by universal sequencing of cancer-related genes in tumor and normal DNA vs guideline-based germline testing. JAMA 2017;318:825-835.

30. Meric-Bernstam F, Brusco L, Daniels M, et al. Incidental germline variants in 1000 advanced cancers on a prospective somatic genomic profiling protocol. Ann Oncol 2016;27:795-800.

31. Seifert BA, O'Daniel JM, Amin K, et al. Germline analysis from tumorgermline sequencing dyads to identify clinically actionable secondary findings. Clin Cancer Res 2016;22:4087-4094.

32. Huang KL, Mashl RJ, Wu Y, et al. Pathogenic germline variants in 10,389 adult cancers. Cell 2018;173:355-370.e14.

33. Abida W, Armenia J, Gopalan A, et al. Prospective genomic profiling of prostate cancer across disease states reveals germline and somatic alterations that may affect clinical decision making. JCO Precis Oncol 2017;2017:PO.17.00029.
34. Carlo MI, Mukherjee S, Mandelker D, et al. Prevalence of germline mutations in cancer susceptibility genes in patients with advanced renal cell carcinoma. JAMA Oncol 2018;4:1228-1235.

35. Carlo MI, Ravichandran V, Srinavasan P, et al. Cancer susceptibility mutations in patients with urothelial malignancies. J Clin Oncol 2020;38:406-414.

36. Whitworth J, Smith PS, Martin JE, et al. Comprehensive cancerpredisposition gene testing in an adult multiple primary tumor series shows a broad range of deleterious variants and atypical tumor phenotypes. Am J Hum Genet 2018;103:3-18.

37. Cadoo K, Mukherjee $S$, Khurram A, et al. Characterization of patients with multiple primary tumors [abstract]. J Clin Oncol 2020;38(Suppl): Abstract 1502.

38. Raj N, Shah R, Stadler Z, et al. Real-time genomic characterization of metastatic pancreatic neuroendocrine tumors has prognostic implications and identifies potential germline actionability. JCO Precis Oncol;2018:PO.17.00267.

39. Latham A, Srinivasan $P$, Kemel $Y$, et al. Microsatellite instability is associated with the presence of Lynch syndrome pan-cancer. J Clin Oncol 2019;37:286-295.

40. Lowery MA, Wong W, Jordan EJ, et al. Prospective evaluation of germline alterations in patients with exocrine pancreatic neoplasms. J Natl Cancer Inst 2018;110:1067-1074.

41. Salo-Mullen EE, O'Reilly EM, Kelsen DP, et al. Identification of germline genetic mutations in patients with pancreatic cancer. Cancer 2015;121:4382-4388.

42. Golan T, Hammel P, Reni M, et al. Maintenance olaparib for germline BRCA-mutated metastatic pancreatic cancer. N Engl J Med 2019;381:317-327.

43. Jonsson $\mathrm{P}$, Bandlamudi $\mathrm{C}$, Cheng ML, et al. Tumour lineage shapes BRCA-mediated phenotypes. Nature 2019;571:576-579.

44. Forsberg LA, Gisselsson D, Dumanski JP. Mosaicism in health and disease_clones picking up speed. Nat Rev Genet 2017;18:128-142.

45. Ptashkin RN, Mandelker DL, Coombs CC, et al. Prevalence of clonal hematopoiesis mutations in tumor-only clinical genomic profiling of solid tumors. JAMA Oncol 2018;4:1589-1593.

46. Lee J, Gubernick LR, Brodsky AL, et al. Missed opportunities: genetic counseling and testing among an ethnically diverse cohort of women with endometrial cancer. Gynecol Oncol 2018;151:153-158.

47. Swisher EM, Rayes N, Bowen D, et al. Results from MAGENTA: a national randomized four-arm noninferiority trial evaluating pre- and post-test genetic counseling during online testing for breast and ovarian cancer genetic risk [abstract]. J Clin Oncol 2020;38(Suppl):Abstract 1506.

48. Erlich $Y$, Narayanan A. Routes for breaching and protecting genetic privacy. Nat Rev Genet 2014;15:409-421.

49. Smith JD, Birkeland AC, Goldman EB, et al. Immortal life of the common rule: ethics, consent, and the future of cancer research. J Clin Oncol 2017;35:1879-1883.

50. Sekulic A, Migden MR, Oro AE, et al. Efficacy and safety of vismodegib in advanced basal-cell carcinoma. N Engl J Med 2012;366:2171-2179.

51. Wells SA Jr, Robinson BG, Gagel RF, et al. Vandetanib in patients with locally advanced or metastatic medullary thyroid cancer: a randomized, double-blind phase III trial. J Clin Oncol 2012;30:134-141.

52. Wirth LJ, Sherman E, Robinson B, et al. Efficacy of selpercatinib in RETaltered thyroid cancers. N Engl J Med 2020;383:825-835.

53. Bissler JJ, Kingswood JC, Radzikowska E, et al. Everolimus for angiomyolipoma associated with tuberous sclerosis complex or sporadic lymphangioleiomyomatosis (EXIST-2): a multicentre, randomised, double-blind, placebo-controlled trial. Lancet 2013;381:817-824.

54. Dombi E, Baldwin A, Marcus LJ, et al. Activity of selumetinib in neurofibromatosis type 1-related plexiform neurofibromas. N Engl J Med 2016;375:2550-2560.

55. Krueger DA, Care MM, Holland K, et al. Everolimus for subependymal giant-cell astrocytomas in tuberous sclerosis. N Engl J Med 2010;363:1801-1811.

56. Le DT, Durham JN, Smith KN, et al. Mismatch repair deficiency predicts response of solid tumors to PD-1 blockade. Science 2017;357:409-413.

57. Domchek SM, Mardis E, Carlisle JW, et al. Integrating genetic and genomic testing into oncology practice. Am Soc Clin Oncol Educ Book 2020;40:e259-263.

58. Moore K, Colombo N, Scambia G, et al. Maintenance olaparib in patients with newly diagnosed advanced ovarian cancer. N Engl J Med 2018;379:2495-2505. 
59. Swisher EM, Lin KK, Oza AM, et al. Rucaparib in relapsed, platinumsensitive high-grade ovarian carcinoma (ARIEL2 part 1): an international, multicentre, open-label, phase 2 trial. Lancet Oncol 2017;18:75-87.

60. Ray-Coquard IL, Pautier P, Pignata S, et al. Phase III PAOLA-1/ENGOTov25 trial: olaparib plus bevacizumab (bev) as maintenance therapy in patients (pts) with newly diagnosed, advanced ovarian cancer (OC) treated with platinum-based chemotherapy (PCh) plus bev [abstract]. Ann Oncol 2019;30(Suppl 5):v851-934. Abstract LBA2_PR.

61. Moore KN, Secord AA, Geller MA, et al. Niraparib monotherapy for lateline treatment of ovarian cancer (QUADRA): a multicentre, open-label, single-arm, phase 2 trial. Lancet Oncol 2019;20:636-648.

62. González-Martín A, Pothuri B, Vergote I, et al. Niraparib in patients with newly diagnosed advanced ovarian cancer. N Engl J Med 2019;381:2391-2402.

63. Litton JK, Rugo HS, Ettl J, et al. Talazoparib in patients with advanced breast cancer and a germline BRCA mutation. N Engl J Med 2018;379:753-763.

64. Robson M, Im SA, Senkus E, et al. Olaparib for metastatic breast cancer in patients with a germline BRCA mutation. $\mathrm{N}$ Engl J Med 2017;377:523-533.

65. Abida W, Patnaik A, Campbell D, et al. Rucaparib in men with metastatic castration-resistant prostate cancer harboring a BRCA1 or BRCA2 gene alteration. J Clin Oncol 2020;38:3763-3772.
66. de Bono J, Mateo J, Fizazi K, et al. Olaparib for metastatic castrationresistant prostate cancer. N Engl J Med 2020;382:2091-2102.

67. Domchek SM, Aghajanian C, Shapira-Frommer R, et al. Efficacy and safety of olaparib monotherapy in germline BRCA1/2 mutation carriers with advanced ovarian cancer and three or more lines of prior therapy. Gynecol Oncol 2016;140:199-203.

68. Thavaneswaran S, Rath E, Tucker K, et al. Therapeutic implications of germline genetic findings in cancer. Nat Rev Clin Oncol 2019;16:386-396.

69. Stadler ZK, Maio A, Chakravarty D, et al. Therapeutic implications of germline testing in patients with advanced cancers [published online June 16, 2021]. J Clin Oncol, doi: 10.1200/JCO.20.03661.

70. Tung NM, Boughey JC, Pierce LJ, et al. Management of hereditary breast cancer: American Society of Clinical Oncology, American Society for Radiation Oncology, and Society of Surgical Oncology guideline. J Clin Oncol 2020;38:2080-2106.

71. Litton JK, Scoggins ME, Hess KR, et al. Neoadjuvant talazoparib for patients with operable breast cancer with a germline BRCA pathogenic variant. J Clin Oncol 2020;38:388-394.

72. Stjepanovic N, Stockley TL, Bedard PL, et al. Additional germline findings from a tumor profiling program. BMC Med Genomics 2018;11:65.

73. Offit K, Tkachuk KA, Stadler ZK, et al. Cascading after peridiagnostic cancer genetic testing: an alternative to population-based screening. J Clin Oncol 2020;38:1398-1408. 